\title{
Adaptability, Cognitive Flexibility, Personal Need for Structure, and Rigidity*
}

\author{
Armanda Hamtiaux, Claude Houssemand \\ University of Luxembourg, Walferdange, Luxembourg
}

\begin{abstract}
IA (individual adaptability), COFL (cognitive flexibility), PNS (personal need for structure), and rigidity of attitudes, all have an aim in common to evaluate the person's self-perceived capacity to manifest a flexible or inflexible behavior in a given situation. However, these seemingly related concepts have rarely been investigated jointly. The goal of the present research is twofold: (1) to explore elements of discriminate and convergent validity of IA by relating it to COFL, PNS, and rigidity; and (2) to examine individual differences regarding gender, educational attainment, and labor force status in regards to the previously mentioned concepts. The results suggest that these concepts are related but remain distinct constructs, and that they differ in their capacity to differentiate between individuals based on gender, education, and labor force status.
\end{abstract}

Keywords: adaptability, COFL (cognitive flexibility), PNS (personal need for structure), rigidity, differential psychology

\section{Introduction}

The question of whether a person is adaptable or not and whether a person is able to adjust to new or changing situations (Morrison \& Hall, 2002) is becoming more and more important in our rapidly evolving private and professional world. Consequently, there has been an increase in the number of articles published on this particular issue. Even though the topic has been addressed frequently in the scientific literature, most of the published articles treat a specific aspect of it, such as personal adaptability at work (O'Connell, Mc Neely, \& Hall, 2008), career (Savickas et al., 2009), or IA (individual adaptability) (Ployhart \& Bliese, 2006); or explore its role in a given context (Tucker, Pleban, \& Gunther, 2010).

In regard to previously published work (Lang \& Bliese, 2009; Morrison \& Hall, 2002; O'Connell et al., 2008; Ployhart \& Bliese, 2006; Savickas, 1997; Savickas et al., 2009), the concept is still lacking consensus in its definition and elements that make up adaptability, and still needs to be identified and clarified. In response to this lack of identification, the current study attempts to provide some insight about IA (Ployhart \& Bliese, 2006) by relating it to conceptually similar constructs, such as RAPH (rigidity of attitudes regarding personal habits) (Meresko, Rubin, Shontz, \& Morrow, 1954), PNS (personal need for structure) (Neuberg \& Newsom, 1993), and COFL (cognitive flexibility) (Martin \& Rubin, 1995).

\footnotetext{
*Acknowledgements: The authors are grateful to Dr. Steinmetz Jean-Paul for providing valuable comments and suggestions during the preparation of the manuscript as well as for providing the French version of the scales. The authors also thank Dr. Salvador Rivas for carefully proofreading the document.

Armanda Hamtiaux, Ph.D., Centre for Educational Measurement and Applied Cognitive Science, University of Luxemburg.

Claude Houssemand, Ph.D., professor, Centre for Educational Measurement and Applied Cognitive Science, University of Luxemburg.
} 
What these concepts and IA have in common is that they evaluate the person's self-perceived capacity to manifest a flexible or inflexible behavior or attitude in a given situation. As such, rigidity of attitudes, PNS, and COFL are thought to be good indicators of elements associated with adaptability. Note that besides a remarkable literature review (Schultz \& Searleman, 2002) and a more recent study (Steinmetz, Loarer, \& Houssemand, 2011), investigations around rigidity mostly stem from the mid-20th century. During that same period, research concerned with adaptability has also been undertaken (Trites, 1960; Trites, Kubala, \& Cobb, 1959), but these seemingly related concepts have never been juxtaposed. Consequently, the authors aim to examine to what extent IA is associated with the previously mentioned constructs. Furthermore, individual differences based on gender, educational attainment, and the labor force statuses of participants are addressed.

\section{Adaptability, COFL, PNS, and Rigidity}

Ployhart and Bliese (2006) used their I-ADAPT model to define IA as an eight-dimensional concept that is influenced and determined to some extent by different KSAOs (knowledge, skills, abilities, and others). These KSAOs represent personality, cognitive abilities, interests, values, physical aptitudes, and so on. In Ployhart and Bliese's (2006) model, IA is influenced by KSAOs and itself is a mediator between the latter concepts and performance (Tucker et al., 2010). Following Pulakos, Arad, Donovan, and Plamodon (2000), Pulakos, Schmitt, Dorsey, Arad, Borman, and Hedge (2002), and Pulakos, Dorsey, and White (2006), IA is defined as an eight-dimensional construct and dimensions are labeled as follows: crisis, culture, creativity, interpersonal, learning, work stress, physical, and uncertainty (see Appendix). A previous study provided support for the theoretically defined, multi-dimensional structure of IA (by current authors) and reported on the inter-relatedness of IA with career (Savickas et al., 2009) and personal adaptability (O'Connell et al., 2008). The major outcomes of that study suggest that IA is indeed an eight-dimensional construct that, compared to the other scales, covers specific aspects of adaptability as well as the general tendency of the person's capacity to adapt.

According to Steinmetz, Loarer, and Houssemand (2011), the rigid individual can be described as a person demonstrating an inability to adjust his/her behavior to a changing environment. Consequently, rigidity of attitudes would appear to be associated with conduct opposite to adaptable behavior. Although it is tempting to see rigidity and adaptability on one continuum, with rigidity on one end and adaptability on the other, it would not be as straight-forward because both adaptability and rigidity are defined as multidimensional constructs (Ployhart \& Bliese, 2006; Steinmetz et al., 2011). Steinmetz et al. (2011) reported elements of construct validity for rigidity using PNS, COFL, and two personality traits: openness and conscientiousness. Because rigidity is differentially related to these concepts, it is understood as having different dimensions. Based on these findings, the rigid person can be characterized as rather close-minded and less open to new experiences and appears to have a strong desire to restructure his/her environment. In contrast, the adaptable person could be described as an individual having facilities in dealing with new situations, including situations that are neither well-structured nor well-known, thus uncertain and unpredictable. Following these descriptions, it becomes obvious that adaptability can be considered in opposition to PNS, a concept that is strongly related to rigidity of attitudes.

PNS is composed of two subscales consisting of the person's desire to have a structured environment, and the person's response to lack of structure in a given situation. Steinmetz et al. (2011) showed that there is a stronger relation between the rigidity scale and the "desire for structure" component than with the "response to 
lack of structure" component. As it can be drawn from the corresponding descriptions, the first component is more about the "need"- the personal desire the person has to be in a well-defined, well-structured, and known environment; whereas the second component is more about the "reaction"- the response to an unstructured and uncertain environment. A major difference between the first component of PNS and adaptability is that the first one evaluates a need or desire of a person, whereas adaptability is concerned with the self-evaluated capacity. The second component is closer to adaptability as it deals with the possible response to a lack of structure, which is more about how people "think" they deal with an unstable environment.

As such, the stronger the person's need for structure, the less adaptable or flexible he/she might be. It seems quite obvious that a more adaptable person should also be more flexible. In corroboration with this point, Steinmetz et al. (2011) used COFL as an opposite measure of rigidity. COFL (Martin \& Rubin, 1995) is seen as the person's awareness that various situations and options may exist, the person's willingness to be flexible and adapt, and the person's belief about their self-efficacy in being flexible. Cognitively speaking, a flexible person would be aware of the existence of different situations and he/she would be willing to adjust to the given situation according to his/her self-evaluated efficacy. Consequently, persons that perceive themselves as cognitively flexible should evaluate themselves as adaptable as well. Note that COFL as it is evaluated here, is neither to be confused with Kobasa's (1979) COFL (defined as a component of personal hardiness that describes the person as perceiving change as a challenge or as an opportunity for further development), which has been used in Pulakos et al. (2006) as a possible predictor of adaptive performance, nor with COFL as evaluated with dynamic complex problem-solving tasks (Cañas, Quesada, Antolí, \& Fajardo, 2003).

\section{The Present Study}

The present study explores elements of discriminant and convergent validity for IA (Ployhart \& Bliese, 2006). It aims for a better understanding of IA by relating it to RAPH and COFL. First, relationships between the different constructs are explored through correlational analyses (correlations and regressions). Second, individual differences regarding gender, educational attainment, and the labor force status (i.e., student, professional, or homemaker) are examined.

\section{Hypotheses}

\section{Overall IA and COFL}

The authors expect a positive relationship between IA and COFL. The authors suggest that overall IA is positively related to COFL, because both concepts refer to a competence or capacity to deal with changing and/or new situations. Both are concerned with the flexibility or the adaptability, which a person can or will demonstrate under certain circumstances. Both assess the person's willingness and his/her self-evaluated efficacy about dealing with situations requiring flexibility and/or adaptability. Thus, the authors expect a strong relationship between both scales.

\section{IA Dimensions and COFL ${ }^{1}$}

At the dimensional level, the authors hypothesize differential relationships between the eight IA dimensions and COFL. More precisely, the authors hypothesize a strong positive relation between COFL and

\footnotetext{
${ }^{1}$ Even though we refer to cognitive elements or processes, it is important to note that in the current research only self-assessment instruments were used, and thus, it is impossible to have any indication of the subject's real cognitive abilities. We have to rely on the self-perceived and self-reported competence of the persons.
} 
the creativity dimension of IA as both measures involve the capacity to solve problems in a creative way and draw on a cognitive component. According to Martin and Rubin (1995), the COFL scale evaluates the person's awareness that different situations or options can take place or occur. This awareness refers to a cognitive capacity or ability to appropriately interpret a situation. Furthermore, they stated that a person that is: (1) aware of different options and must also be willing; (2) to act flexibly in the situation and must believe that he/she can control his/her behavior (self-efficacy); and (3) able to provide an appropriate response to the requirements of the situation. The authors expect the three elements composing COFL are part of all adaptability dimensions, and therefore suggest that all dimensions are related to that concept. The stronger relation between COFL and the creativity dimensions of IA is expected because of content similarities between both item groups.

\section{IA and PNS}

The authors expect to find a negative and weak relationship between IA and PNS. The relation is expected to be negative, because adaptability is mainly concerned with handling unknown, new, or unpredictable situations; whereas PNS refers to a person's desire for known and well-structured environments or the response to a lack of structure in the environment. Therefore, a person who perceives himself/herself as having good competences in dealing with novelty and uncertainty is expected to attribute himself/herself lower scores on the desire for structure and the response to lack of structure components. Furthermore, the authors expect a stronger relation between IA and the response to lack of structure component, because adaptability items refer to situations presenting lack of structure and do not evaluate the person's desire for certain circumstances.

Finally, the authors expect all correlations to be low, because IA and PNS are supposed to be related, but they are different constructs nevertheless. They believe the way IA is assessed here provides an indication how adaptable the person believes to be in different situations and contexts, e.g., crisis or uncertain situations, or in human interaction situations. PNS, however, appears to be more specific in its evaluation and in asking about the person's position toward well-organized daily situations. As such, a more specific aspect of the PNS (i.e., routine) is evaluated. Consequently, the authors expect both dimensions of PNS to be more strongly related to the uncertainty dimension of IA. Moreover, if it is considered that people with a high need for structure prefer a routinized systematic environment, then it is expected that these people dislike changing working conditions, and therefore, also a stronger relation between PNS and the work stress dimension of IA is expected.

\section{IA and RAPH}

The authors expect IA and rigidity of attitudes to be negatively associated. Definition rigidity and adaptability are opposite concepts. Steinmetz et al. (2011) showed that rigidity and PNS were related $(r=0.57$, $p<0.05 ; N=204)$. As the authors hypothesize a negative relationship between adaptability and PNS, they also expect a negative relation between adaptability and rigidity. Moreover, the authors do not expect strong correlations between IA and rigidity of attitudes because they are considered to be different concepts. As the rigidity scale is more about the individual's self-perceived attitudes and habits in regard to rigid behavior, the highest correlations are expected with the uncertainty dimension and the work stress dimension of IA.

\section{Method}

\section{Participants}

Data were collected from a sample of 83 subjects ( 50 women and 33 men; mean age 38.7 years old, $S D=$ 12.3; Med. =26). Participants were recruited via students who had to take part in a research project for course credit. Before collecting data among their peers, friends, and family members, students received explanation 
about the different concepts and were informed about how to reliably collect data. All participants replied to a set of socio-demographic questions and completed a set of selected measures hypothesized to be related with IA. Fifty-nine percent of the participants were of Luxemburgish nationality, $12 \%$ were German, 9.6\% were Portuguese, 2.4\% were French, $15.7 \%$ had another nationality, and $1.2 \%$ did not specify. Concerning educational attainment, $17.7 \%$ had less than a high-school degree, $18.1 \%$ possessed a high school degree, $22.9 \%$ a bachelor's degree, and $31.3 \%$ had a master's degree or higher. Sixty-nine percent were professionals, $20.5 \%$ were students, $13.2 \%$ were not employed (i.e., homemaker, retired, or other), and $2.4 \%$ did not provide relevant information. Mean number of years of work experience was 13.9 years $(S D=12.8$; Med $=11)$, but $15.7 \%$ had no work experience at all. All participants completed a French-translated version of IA and the three measures of convergent and divergent concepts ${ }^{2}$.

\section{Instruments}

IA $^{3}$. A French version of the eight-dimensional IA scale composed of 55 items was used (Ployhart \& Bliese, 2006). Results for only two of the eight dimensions have been published previously. Wessel, Ryan, and Oswald (2008) used the English version of the learning and uncertainty dimensions and reported an alpha of $0.79(N=198)$ for the learning adaptability dimension and an alpha of $0.74(N=198)$ for the uncertainty dimension. The candidates responded using a 5-point Likert-scale ranging from "Strongly disagree" to "Strongly agree". Examples of items are: "I usually over-react to stressful news" (work stress dimension) and "I take responsibility for acquiring new skills" (learning dimension).

COFL (Congruent measure: Cognitive flexibility). The COFL scale (Martin \& Rubin, 1995) is a 12-item scale. In its original version, the scale demonstrated acceptable internal consistency with an alpha around 0.77 (Martin \& Rubin, 1995). Response options in the questionnaire ranged from "Strongly agree" to "Strongly disagree" on a 6-point Likert-scale. Examples of items are: "In any given situation, I am able to act appropriately" and "I avoid new and unusual situations".

RAPH (Divergent measure: Rigidity of attitudes regarding personal habits). Rigidity was assessed with RAPH scale (Meresko et al., 1954). Originally, the scale was designed to evaluate the person's opposition to change and intolerance of ambiguity. The scale comprised 20 items and response options ranged from "Strongly disagree" to "Strongly agree" on a 7-point Likert-scale. Steinmetz et al. (2011) reported acceptable internal consistency with an alpha being $0.81(N=204)$. An example of an item is: "I dislike doing anything just on the spur of the moment".

PNS (Divergent measure: Personal need for structure). PNS (Neuberg \& Newsom, 1993) was evaluated with the PNS scale incorporating two main components: "desire for structure" (4 items) and "response to lack of structure" (7 items). The scale consisted of 11 items and required the candidates to answer using a 6-point Likert-scale, with response options ranging from "Strongly disagree" to "Strongly agree". Even though the original scale integrated 12 items, the authors followed Neuberg and Newsom's (1993) recommendation and omitted Item 5 because of its possible misinterpretation. Neuberg and Newsom (1993) reported different values for internal consistency, alpha ranging from 0.72 to 0.78 for the "desire for structure" component and ranging from 0.69 to 0.82 for the "response to lack of structure" component with differing sample sizes (from $N=205$ to $N=914$ ). Examples of items are: "I enjoy having a clear and structured mode of

\footnotetext{
${ }^{2}$ For translated versions refer to Steinmetz et al., 2011.

${ }^{3}$ The IA scale (Ployhart \& Bliese, 2006) was translated from English into French via several native French speakers and has been reviewed and verified by different quadrilingual-speaking persons (German, French, English, and Luxemburgish).
} 
life" and "I don't like situations that are uncertain".

\section{Results}

\section{Reliability Analysis}

Cronbach's alphas (see Table 1) are reported here to give an indication of the reliability of the scales the authors investigated. The IA scale, consisting of 55 items, had good reliability $(\alpha=0.91)$. The corresponding reliabilities for the various dimensions of IA ranged from $\alpha=0.72$ (uncertainty) to $\alpha=0.81$ (learning); but the interpersonal and the physical adaptability dimensions seemed to include more heterogeneous items and had relatively low reliabilities ( $\alpha=0.56$ and $\alpha=0.45$, respectively). Previous results (by current authors) have shown that the physical adaptability dimension has relatively low reliability because it appears to be composed of three groups of items. These groups concern the physical state of the person (tiredness), environmental physical conditions (e.g., heat), and physically demanding tasks. In regards to the interpersonal dimension, the aforementioned reliability reported in the previous study was acceptable $(\alpha=0.66)$.

The COFL scale and RAPH scale had good reliability indices with alpha values of 0.75 and 0.83 , respectively. The PNS scale had an alpha value of 0.62 for the "desire for structure" component and an alpha value of 0.73 for the "response to lack of structure" component.

\section{Exploring Relationships_-Correlational Analyses}

Note that the authors draw upon Cohen’s (1992) criteria for medium $( \pm 0.30)$ and large $( \pm 0.50)$ effects for correlation coefficients.

Table 1

Correlational Analysis for IA, COFL, PNS, and Rigidity

\begin{tabular}{|c|c|c|c|c|c|c|c|c|c|c|c|c|c|c|}
\hline & \multicolumn{9}{|c|}{ IA scale and dimension } & \multicolumn{5}{|c|}{ Scale } \\
\hline & IA & Crisis & Culture & $\begin{array}{l}\text { Work } \\
\text { stress }\end{array}$ & Inter-personal & Learning & Physical & Creativity & Uncertainty & RAPH & PNS_D & PNS_R & PNS & COFL \\
\hline IA & $\begin{array}{c}0.91 \\
(55)\end{array}$ & & & & & & & & & & & & & \\
\hline Crisis & $0.78^{* *}$ & $0.79(6)$ & & & & & & & & & & & & \\
\hline Culture & $0.64^{* *}$ & $0.46^{* *}$ & $0.79(5)$ & & & & & & & & & & & \\
\hline Work stress & $0.70^{* *}$ & $0.59^{* *}$ & $0.23^{* *}$ & $0.78(5)$ & & & & & & & & & & \\
\hline $\begin{array}{l}\text { Inter- } \\
\text { personal }\end{array}$ & $0.56^{* *}$ & $0.30^{* *}$ & $0.51^{* *}$ & 0.09 & $0.56(7)$ & & & & & & & & & \\
\hline Learning & $0.62^{* *}$ & $0.34^{* *}$ & $0.32^{* *}$ & $0.24^{*}$ & $0.50^{* *}$ & $0.81(9)$ & & & & & & & & \\
\hline Physical & $0.65^{* *}$ & $0.48^{* *}$ & $0.45^{* *}$ & $0.43^{* *}$ & $0.22^{* *}$ & 0.11 & $0.45(9)$ & & & & & & & \\
\hline Creativity & $0.74^{* *}$ & $0.55^{* *}$ & $0.35^{* *}$ & $0.41^{* *}$ & $0.47^{* *}$ & $0.48^{* *}$ & $0.44^{* *}$ & $0.72(5)$ & & & & & & \\
\hline Uncertainty & $0.77^{* *}$ & $0.54^{* *}$ & $0.39^{* *}$ & $0.59^{* *}$ & $0.30^{* *}$ & $0.34^{* *}$ & $0.44^{* *}$ & $0.45^{* *}$ & $0.72(9)$ & & & & & \\
\hline RAPH & $-0.23^{* *}$ & -0.15 & $-0.28^{* *}$ & $-0.32^{* *}$ & 0.08 & 0.01 & -0.15 & -0.05 & $-0.31^{* *}$ & $\begin{array}{c}0.83 \\
(20)\end{array}$ & & & & \\
\hline PNS_D & $-0.30^{* *}$ & -0.20 & $-0.23^{*}$ & $-0.35^{* *}$ & 0.08 & -0.04 & $-0.25^{*}$ & -0.09 & $-0.41^{* *}$ & $0.60^{* *}$ & $0.62(4)$ & & & \\
\hline PNS_R & $-0.33^{* *}$ & -0.07 & $-0.32^{* *}$ & $-0.32^{* *}$ & -0.09 & $-0.24^{*}$ & $-0.22^{*}$ & -0.16 & $-0.29^{* *}$ & $0.58^{* *}$ & $0.57^{* *}$ & $0.73(7)$ & & \\
\hline PNS & -0.35 & -0.14 & $-0.32^{* *}$ & $-0.37^{* *}$ & -0.03 & -0.18 & $-0.26^{*}$ & -0.15 & $-0.38^{* *}$ & $0.66^{* *}$ & $0.83^{* *}$ & $0.93^{* *}$ & $\begin{array}{c}0.80 \\
(11)\end{array}$ & \\
\hline COFL & $0.65^{* *}$ & $0.49^{* *}$ & $0.40^{* *}$ & $0.39^{* *}$ & $0.51^{* *}$ & $0.44^{* *}$ & $0.37^{* *}$ & $0.57^{* *}$ & $0.45^{* *}$ & -0.04 & -0.10 & -0.17 & -0.16 & $\begin{array}{l}0.75 \\
(12)\end{array}$ \\
\hline
\end{tabular}

Notes. Cronbach's alpha is reported in the diagonal; number of items for the different scales is in parenthesis ()$;{ }^{* *} p<0.01,{ }^{*} p<$ 0.05; COFL: cognitive flexibility, PNS_D: desire for structure of personal need for structure scale, PNS_R: response to lack of structure of personal need for structure scale, RAPH: rigidity of attitudes regarding personal habits, IA: individual adaptability. 


\section{IA and COFL}

The authors hypothesized a strong positive relationship between COFL and overall IA. Results reported in Table 1 show that this hypothesis was confirmed by a strong and positive correlation $(r=0.65, p<0.01)$ suggesting that both scales reflected similar matters/issues. Moreover, in agreement with our prediction, the strongest relation was observed between the person's reported capacity to flexibly deal with problems and the creativity dimension of IA $(r=0.57, p<0.01)$. The other dimensions were also associated with the COFL, correlations ranged from $r=0.37, p<0.01$ with the physical dimension to $r=0.51, p<0.01$ with the interpersonal dimension. The $t$-test statistic based on Chen and Popovich (2002) was used to compare correlations and detect if correlations were significantly different for the various IA dimensions with the flexibility construct. Even though the link between the creativity dimensions and COFL is apparently stronger than with other IA dimensions, the significance test does not support the hypothesis that the creativity dimension has more in common with the COFL of the person than the other dimensions (except for the physical dimension, $\Delta r=0.20, p<0.05$ ). Nevertheless, the correlational analysis between COFL and adaptability provided support for convergent validity.

\section{IA and PNS}

The authors postulated that adaptability is weakly related to PNS and that correlations would be negative. The response to lack of structure component was expected to show a stronger relation with adaptability than the desire for structure component. Moreover, the authors expected the uncertainty and work stress dimensions to be more strongly associated with both dimensions of PNS than the other IA dimensions. The correlational coefficients reported in Table 1, confirm the negative association between the scales and reveal a slightly stronger relation between overall adaptability and the second component of PNS $(r=-0.33, p<0.01 v s . r=$ $-0.30, p<0.01)$. This difference, however, was not statistically significant according to the $t$-test statistic as suggested by Chen and Popovich (2002). Otherwise, the authors observed a similar pattern between the different dimensions of IA and both components of the PNS scale. Only the uncertainty dimension appeared to relate more strongly with the desire component than with the response to lack of structure component; but again correlations did not differ significantly $(\Delta r=0.11, n s)$. Note that none of the differences between the correlations for both components were significant.

In regard to our more specific hypotheses that both components of PNS would be more strongly related to the work stress and uncertainty dimension of IA compared to the other IA dimensions, the results appear to support this assertion at first glance - the most important correlations, for the desire component, are observed with the uncertainty and work stress dimensions $(r=-0.41, p<0.01$ and $r=-0.35, p<0.01$, respectively). However, these values are significantly different $(p<0.05)$ only from the learning $(r=-0.04, n s)$ and creativity $(r=-0.09, n s)$ dimensions for the work stress dimension; and from the crisis $(r=-0.20, n s)$, creativity $(r=-0.09$, $n s)$, interpersonal $(r=0.08, n s)$, and learning $(r=-0.04, n s)$ dimensions $(p<0.01)$ for the uncertainty dimension. In regard to the response to lack of structure, the uncertainty $(r=-0.29, p<0.01)$ and work stress $(r$ $=-0.32, p<0.01)$ dimensions are significantly more strongly related to response to lack of structure component only compared to the crisis $(r=-0.07, n s)$ dimension of IA. Results also revealed that PNS appeared to share very little variance with the creativity and interpersonal dimensions. These zero order correlations also point to the fact that PNS does not tap the same information as the COFL scale, which was highly correlated with these two dimensions. All in all, these results supported the hypothesis of divergent validity with the PNS scale. 


\section{IA and RAPH}

As the definitions for IA and rigidity seem to be in direct contrast, the relation between both concepts was expected to be weak and negative. In support of this, correlational analyses resulted in the expected direction. The effect sizes stayed in the range from low to medium (similar results than for the PNS construct). Overall, adaptability and rigidity were related to each other, but to a lower extent $(r=-0.23, p<0.05)$ than rigidity with the culture, crisis, and uncertainty dimensions of the IA, which were not significant differences (all $\Delta r, n s)$. In regard to the work stress $(r=-0.32, p<0.01)$ and the uncertainty $(r=-0.31, p<0.01)$, these dimensions were significantly stronger related to rigidity than creativity $(r=-0.05, n s)$ or the learning dimension $(r=0.01, n s)$.

To summarize, the correlational analyses provided support for the expected inter-relatedness of the different scales. All four scales, IA, COFL, rigidity, and PNS were related to some extent and in the expected directions. In general, the strongest relation was found between IA and COFL $(r=0.65, p<0.01)$, and the weakest was found between adaptability and rigidity $(r=-0.23, p<0.05)$. As all the correlations appeared to be significant, even though they differed in their strengths, the authors may state that elements in support of convergent and divergent validity were provided.

\section{Exploring Relationships — Linear Regression}

The correlational analyses revealed that COFL, rigidity of attitudes, and PNS were related to IA. Linear regression using a stepwise procedure was used to explore which of the three constructs shares the most variance with IA. This effort was not aimed at establishing a causal relationship, but it was undertaken to provide a better understanding of the links tying these concepts together.

The results in Table 2 show that COFL was retained as a single predictor and accounted from $20 \%$ to $33 \%$ of the variance in the crisis, creativity, interpersonal, and learning dimensions of IA. The cultural dimension involved mainly COFL accounting for $16 \%$ of the variance, but rigidity accounted for an additional $7 \%$ of the variance. The work stress, physical, and uncertainty dimensions were most importantly related to COFL, but additional variance could be accounted for by the desire for structure component of the PNS scale. COFL and desire for structure accounted for $16 \%$ of the variance in the physical adaptability dimension, $23 \%$ in the work stress dimension and as much as $31 \%$ in the uncertainty dimension of the adaptability scale. Forty-seven percent of the variance was accounted for COFL and the desire for structure component, but only $5 \%$ of the variance could be additionally accounted for the desire for structure component, indicating that IA shares an important part of common information with COFL. The uncertainty and the work stress dimensions seemed to involve the desire for structure component more strongly $\left(\Delta R^{2}=0.13\right.$ and $\Delta R^{2}=0.10$, respectively).

Results demonstrate that COFL was most importantly related to the crisis, interpersonal, learning, and creativity dimensions of adaptability. The relation between the capacity people attribute themselves to dealing with the complex problems and the creativity dimension (i.e., finding innovative ways to deal with problems), and to a lesser extent with the learning dimension (i.e., engage in trainings to stay on the top of the profession), is quite obvious because of the "problem-solving" content that is evaluated. However, it appears that this capacity is also related to how people deal with emergency and interpersonal situations. As such, it seems that the COFL scale evaluates a more general capacity for being flexible, which allows a person to choose between different behavioral patterns in reference to emergency and interpersonal interactions. As mentioned before, COFL appears to be related to all IA dimensions, but for the culture, the physical, the uncertainty, and the work stress dimensions, either PNS or rigidity is involved and can explain additional variance above and beyond COFL. 
Table 2

Hierarchical Regression Analysis for IA and IA Dimensions

\begin{tabular}{|c|c|c|c|c|c|}
\hline Criterion & $\mathrm{B}$ & (SE)B & Beta & $R^{2}$ & $\Delta R^{2}$ \\
\hline \multicolumn{6}{|l|}{ Crisis } \\
\hline \multicolumn{6}{|l|}{ Step 1} \\
\hline COFL & 0.24 & 0.05 & $0.49^{* * *}$ & $0.24^{* * *}$ & \\
\hline \multicolumn{6}{|l|}{ Creativity } \\
\hline \multicolumn{6}{|l|}{ Step 1} \\
\hline COFL & 0.22 & 0.04 & $0.57^{* * *}$ & $0.33^{* * *}$ & \\
\hline \multicolumn{6}{|c|}{ Inter-personal } \\
\hline \multicolumn{6}{|l|}{ Step 1} \\
\hline COFL & 0.19 & 0.04 & $0.51^{* * *}$ & $0.27^{* * *}$ & \\
\hline \multicolumn{6}{|l|}{ Learning } \\
\hline \multicolumn{6}{|l|}{ Step 1} \\
\hline COFL & 0.30 & 0.07 & $0.44^{* * *}$ & $0.20^{* * *}$ & \\
\hline \multicolumn{6}{|l|}{ Culture } \\
\hline \multicolumn{6}{|l|}{ Step 1} \\
\hline COFL & 0.16 & 0.04 & $0.40^{* * *}$ & $0.16^{* * *}$ & \\
\hline \multicolumn{6}{|l|}{ Step 2} \\
\hline COFL & 0.15 & 0.04 & $0.39^{* * *}$ & & \\
\hline RAPH & -0.04 & 0.02 & $-0.26^{* *}$ & $0.23^{* *}$ & $0.07^{* *}$ \\
\hline \multicolumn{6}{|l|}{ Physical } \\
\hline \multicolumn{6}{|l|}{ Step 1} \\
\hline COFL & 0.21 & 0.06 & $0.37^{* *}$ & $0.14^{* *}$ & \\
\hline \multicolumn{6}{|l|}{ Step 2} \\
\hline COFL & 0.20 & 0.06 & $0.35^{* *}$ & & \\
\hline PNS_D & -0.26 & 0.12 & $-0.22^{*}$ & $0.19^{*}$ & $0.05^{*}$ \\
\hline \multicolumn{6}{|c|}{ Uncertainty } \\
\hline \multicolumn{6}{|l|}{ Step 1} \\
\hline COFL & 0.29 & 0.06 & $0.45^{* * *}$ & $0.20^{* * *}$ & \\
\hline \multicolumn{6}{|l|}{ Step 2} \\
\hline COFL & 0.26 & 0.06 & $0.41^{* * *}$ & & \\
\hline PNS_D & -0.49 & 0.12 & $-0.37^{* * *}$ & $0.33^{* * *}$ & $0.13^{* * *}$ \\
\hline \multicolumn{6}{|c|}{ Work stress } \\
\hline \multicolumn{6}{|l|}{ Step 1} \\
\hline COFL & 0.25 & 0.06 & $0.39^{* * *}$ & $0.16^{* * *}$ & \\
\hline \multicolumn{6}{|l|}{ Step 2} \\
\hline COFL & 0.23 & 0.06 & $0.36^{* * *}$ & & \\
\hline PNS_D & -0.42 & 0.13 & $-0.31^{* *}$ & $0.26^{* *}$ & $0.10^{* *}$ \\
\hline \multicolumn{6}{|l|}{ IA } \\
\hline \multicolumn{6}{|l|}{ Step 1} \\
\hline COFL & 1.86 & 0.24 & $0.65^{* * *}$ & $0.42^{* * *}$ & \\
\hline \multicolumn{6}{|l|}{ Step 2} \\
\hline COFL & 1.79 & 0.23 & $0.63^{* * *}$ & & \\
\hline PNS_D & -1.40 & 0.49 & $-0.23^{* *}$ & $0.47^{* *}$ & $0.05^{* *}$ \\
\hline
\end{tabular}

Notes. COFL: cognitive flexibility, RAPH: rigidity, PNS_D: desire for structure of the personal need for structure scale; ${ }^{* * *} p<$ $0.001,{ }^{* *} p<0.01,{ }^{*} p<0.05$. 


\section{Comparing Means}

The authors' goal at the outset was not only to explore the relations between the different concepts, but also to investigate "if" and "how" individual differences could be described with these instruments. Analyzing individual differences using the different instruments should also provide information about how the scales could be used in practical settings, such as in counseling and career development.

The authors compared standardized means for IA, COFL, PNS, and rigidity of attitudes along differences in gender, educational attainment, and the participants' current occupational status. The comparisons took place at two levels: First, the authors analyzed the differences at a composite score level for all four scales with univariate ANOVAs (analysis of variance); Second, they explored differences at the dimensional level for the constructs that measured more than one dimension-The authors compared IA and PNS using MANOVAs (multivariate analysis of variance). This approach was adopted to allow a more accurate comparison between entire scales (ANOVA) without omitting valuable information about the more specific aspects of IA or PNS (MANOVA).

Table 3

Standardized Means (Z-scores) and SD (Standard Deviation) for IA, PNS, COFL, and RAPH in Regard to Gender, Educational Attainment, and Labor Force Status

\begin{tabular}{|c|c|c|c|c|c|c|c|c|c|}
\hline & \multirow{2}{*}{$N$} & \multicolumn{2}{|r|}{ IA } & \multicolumn{2}{|r|}{ PNS } & \multicolumn{2}{|r|}{ COFL } & \multicolumn{2}{|r|}{ RAPH } \\
\hline & & $M(S D)$ & $95 \% \mathrm{CI}$ & $M(S D)$ & $95 \% \mathrm{CI}$ & $M(S D)$ & $95 \% \mathrm{CI}$ & $M(S D)$ & $95 \% \mathrm{CI}$ \\
\hline \multicolumn{10}{|l|}{ Gender } \\
\hline Male & 33 & $\begin{array}{c}0.42 \\
(0.80)\end{array}$ & $(0.10,0.75)$ & $\begin{array}{l}-0.18 \\
(0.99)\end{array}$ & $(-0.53,0.16)$ & $\begin{array}{c}0.41 \\
(0.83)\end{array}$ & $(0.08,0.74)$ & $\begin{array}{l}-0.07 \\
(1.01)\end{array}$ & $(-0.42,0.28)$ \\
\hline Female & 50 & $\begin{array}{l}-0.28 \\
(1.03) \\
\end{array}$ & $(-0.54,-0.01)$ & $\begin{array}{c}0.12 \\
(1.00)\end{array}$ & $(-0.16,0.40)$ & $\begin{array}{l}-0.27 \\
(1.02)\end{array}$ & $(-0.54,-0.01)$ & $\begin{array}{c}0.05 \\
(1.00) \\
\end{array}$ & $(-0.24,0.33)$ \\
\hline \multicolumn{10}{|c|}{ Educational attainment } \\
\hline LHS & 23 & $\begin{array}{l}-0.68 \\
(1.08)\end{array}$ & $(-1.06,-0.30)$ & $\begin{array}{c}0.58 \\
(1.05)\end{array}$ & $(0.19,0.96)$ & $\begin{array}{l}-0.42 \\
(0.98)\end{array}$ & $(-0.82,-0.01)$ & $\begin{array}{c}0.58 \\
(0.89)\end{array}$ & $(0.20,0.95)$ \\
\hline HS & 15 & $\begin{array}{c}0.35 \\
(0.90)\end{array}$ & $(-0.12,0.82)$ & $\begin{array}{c}0.18 \\
(0.65)\end{array}$ & $(-0.29,0.66)$ & $\begin{array}{c}0.30 \\
(1.16)\end{array}$ & $(-0.20,0.80)$ & $\begin{array}{c}0.33 \\
(0.77)\end{array}$ & $(-0.14,0.79)$ \\
\hline BA & 19 & $\begin{array}{c}0.02 \\
(0.77)\end{array}$ & $(-0.39,0.44)$ & $\begin{array}{l}-0.19 \\
(0.86)\end{array}$ & $(-0.62,0.23)$ & $\begin{array}{l}-0.06 \\
(0.69)\end{array}$ & $(-0.51,0.38)$ & $\begin{array}{l}-0.35 \\
(0.88)\end{array}$ & $(-0.77,0.06)$ \\
\hline MA & 26 & $\begin{array}{c}0.38 \\
(0.85)\end{array}$ & $(0.03,0.74)$ & $\begin{array}{l}-0.47 \\
(0.98)\end{array}$ & $(-0.83,-0.11)$ & $\begin{array}{c}0.24 \\
(1.03)\end{array}$ & $(-0.14,0.62)$ & $\begin{array}{l}-0.44 \\
(1.02)\end{array}$ & $(-0.79,-0.08)$ \\
\hline \multicolumn{10}{|c|}{ Labor force status } \\
\hline PLI & 9 & $\begin{array}{l}-1.08 \\
(0.86)\end{array}$ & $(-1.70,-0.45)$ & $\begin{array}{c}0.72 \\
(1.13)\end{array}$ & $(0.07,1.37)$ & $\begin{array}{l}-0.81 \\
(0.71)\end{array}$ & $(-1.44,-0.18)$ & $\begin{array}{c}0.75 \\
(0.97)\end{array}$ & $(0.09,1.40)$ \\
\hline ST & 17 & $\begin{array}{l}-0.14 \\
(0.69)\end{array}$ & $(-0.59,0.32)$ & $\begin{array}{l}-0.21 \\
(1.05)\end{array}$ & $(-0.68,0.27)$ & $\begin{array}{c}0.01 \\
(0.85)\end{array}$ & $(-0.45,0.47)$ & $\begin{array}{l}-0.24 \\
(0.69)\end{array}$ & $(-0.72,0.23)$ \\
\hline PL & 55 & $\begin{array}{c}0.21 \\
(1.01)\end{array}$ & $(-0.04,0.46)$ & $\begin{array}{l}-0.07 \\
(0.94)\end{array}$ & $(-0.33,0.20)$ & $\begin{array}{c}0.18 \\
(1.00)\end{array}$ & $(-0.08,0.43)$ & $\begin{array}{l}-0.04 \\
(1.06)\end{array}$ & $(-0.30,0.22)$ \\
\hline
\end{tabular}

Notes. IA: individual adaptability; PNS: personal need for structure; COFL: cognitive flexibility; RAPH: rigidity of attitudes regarding personal habits; CI: confidence interval; LHS: lower than high school degree; HS: high school degree; BA: bachelor; MA: master degree or more; PLI: retired, unemployed, and homemaker; ST: student; PL: professional active.

Given the inequality of sample sizes, the authors checked Box's M and Levene's test for each of the following analyses in reference to gender, educational attainment, and the person's labor force status. Overall, the authors believe that MANOVA may be used here, as no substantial anomalies have been found neither for Box's M, Levene's test, nor regarding the assumption of normal distribution. Furthermore, Hochberg's statistics were used because of unequal sample size for pairwise comparisons and the Bonferroni adjustment 
was applied to control for accumulation of type I error.

\section{Gender}

To investigate differences between men and women, the authors computed univariate ANOVAs for the composite scores of IA, COFL, PNS, and rigidity of attitudes. Results revealed a significant gender effect on the composite score for overall IA, $F_{(1,81)}=10.96, p<0.01, \eta^{2}=0.119$, power $=0.905$ : $\left(z_{\text {men }}=0.42 v s . z_{\text {women }}=\right.$ -0.28). There was no significant difference regarding PNS, $F_{(1,81)}=1.82$, $n s$, and a non-significant effect of gender on rigidity, $F_{(1,81)}=0.28$, ns. A significant gender effect also appeared for COFL, $F_{(1,81)}=10.40, p<$ $0.01, \eta^{2}=0.114$, power $=0.890:\left(z_{\text {men }}=0.41 v s . z_{\text {women }}=-0.27\right)$. All standardized means and $S D$ are reported in Table 3.

As mentioned earlier, the authors explored IA at a dimensional level by gender using MANOVA. The one-way MANOVA revealed a significant multivariate main effect for gender, Wilk's $\Lambda=0.74, F_{(8,74)}=3.26$, $p<0.01$, partial $\eta^{2}=0.261$. Power to detect the effect was 0.957 . Given the significance of the overall test, the univariate main effects were also examined. Significant univariate main effects for gender were found for crisis, $F_{(1,81)}=4.83, p<0.05$, partial $\eta^{2}=0.056$, power $=0.584:\left(z_{\operatorname{men}}=0.29 v s . z_{\text {women }}=-0.19\right)$; work stress, $F_{(1,81)}=$ 15.33, $p<0.001$, partial $\eta^{2}=0.159$, power $=0.972:\left(z_{\mathrm{men}}=0.49 v s . z_{\mathrm{women}}=-0.32\right)$; physical, $F_{(1,81)}=6.73, p<$ 0.05 , partial $\eta^{2}=0.077$, power $=0.727:\left(z_{\text {men }}=0.34\right.$ vs. $\left.z_{\text {women }}=-0.22\right)$; and creativity, $F_{(1,81)}=13.85, p<0.001$, partial $\eta^{2}=0.146$, power $=0.957:\left(z_{\mathrm{men}}=0.47 v s . z_{\mathrm{women}}=-31\right)$. For all four dimensions, men reported being more adaptable than women.

Moreover, gender accounted for $11.4 \%$ of the variance in COFL and $11.9 \%$ in overall IA (composite score). About $26 \%$ of the variance was explained by gender using MANOVA and relying on the eight more specific dimensions of IA. At a dimensional level, the highest percentage of explained variance for gender was work stress $(15.9 \%)$ followed by the creativity dimension (14.6\%), then the physical and the crisis dimensions (7.7\% and 5.6\%, respectively). Consequently, gender seems to have an important association with how adaptable persons perceive themselves and/or report to be. As such, men evaluate themselves more adaptable than women do and it is especially true for handling work stress situations and being able to find innovative solutions to complex problems.

Even though the authors could not find significant differences with the univariate method regarding PNS, the authors tested for significant differences at the dimensional level. Wilks' $\Lambda=0.97, F_{(2,80)}=1.10, n s$, showed that there was no significant effect of gender on either component of PNS. Descriptive statistics for the dimensions of IA and PNS are reported in Table 4.

\section{Educational Attainment}

Four groups were distinguished based on the participants' reported level of educational attainment: LHS (lower than high school degree) $(N=23)$, HS (high school degree) $(N=15)$, BA (bachelor's degree) $(N=19)$, and MA (master's or higher degree) $(N=26)$. At a general univariate level, significant differences between the four groups of educational attainment were found for IA, $F_{(3,79)}=6.57, p<0.01, \eta^{2}=0.20$, and power = 0.966. At a group level, it appeared that the LHS group was significantly different from the HS group $\left(z_{\mathrm{LHS}}=\right.$ $\left.-0.68 v s . z_{\mathrm{HS}}=0.35\right)$ and from the MA group $\left(z_{\mathrm{LHS}}=-0.68 v s . z_{\mathrm{MA}}=0.38\right)$, but not significantly different from the BA group in regard to self-reported IA. Pairwise comparison also showed that the HS, BA, and MA group did not differ significantly on overall IA. The result revealed that people with a lower level of education tended to evaluate their level of adaptability to be lower than people having an HS or MA degree. 
Even though the difference with the BA group was not significant, all "higher education" groups reported a higher level of adaptability.

Table 4

Standardized Means (Z-scores) and SD of IA Dimensions and PNS Components in Regard to Gender, Educational Attainment, and Labor Force Status

(1)

\begin{tabular}{|c|c|c|c|c|c|c|c|c|c|}
\hline & \multirow[b]{3}{*}{$N$} & \multicolumn{8}{|c|}{ IA } \\
\hline & & \multicolumn{2}{|r|}{ Crisis } & \multicolumn{2}{|r|}{ Culture } & \multicolumn{2}{|c|}{ Work stress } & \multicolumn{2}{|c|}{ Inter-personal } \\
\hline & & $M(S D)$ & $95 \% \mathrm{CI}$ & $M(S D)$ & $95 \% \mathrm{CI}$ & $M(S D)$ & $95 \% \mathrm{CI}$ & $M(S D)$ & $95 \% \mathrm{CI}$ \\
\hline \multicolumn{10}{|l|}{ Gender } \\
\hline Male & 33 & $\begin{array}{c}0.29 \\
(0.95)\end{array}$ & $(-0.05,0.63)$ & $\begin{array}{c}0.05 \\
(1.04)\end{array}$ & $\begin{array}{r}(-0.30 \\
0.40)\end{array}$ & $\begin{array}{c}0.49 \\
(0.74)\end{array}$ & $(0.17,0.81)$ & $\begin{array}{c}0.15 \\
(0.90)\end{array}$ & $(-0.20,0.49)$ \\
\hline Female & 50 & $\begin{array}{l}-0.19 \\
(1.00)\end{array}$ & $(-0.47,0.08)$ & $\begin{array}{l}-0.03 \\
(0.98) \\
\end{array}$ & $\begin{array}{r}(-0.32, \\
0.25) \\
\end{array}$ & $\begin{array}{l}-0.32 \\
(1.02) \\
\end{array}$ & $(-0.58,-0.06)$ & $\begin{array}{l}-0.10 \\
(1.06)\end{array}$ & $(-0.38,0.18)$ \\
\hline \multicolumn{10}{|l|}{$\begin{array}{l}\text { Educational } \\
\text { attainment }\end{array}$} \\
\hline LHS & 23 & $\begin{array}{l}-0.56 \\
(1.22)\end{array}$ & $\begin{array}{r}(-0.96 \\
-0.17)\end{array}$ & $\begin{array}{l}-0.33 \\
(1.11)\end{array}$ & $\begin{array}{r}(-0.74 \\
0.09)\end{array}$ & $\begin{array}{l}-0.80 \\
(1.02)\end{array}$ & $(-1.16,-0.44)$ & $\begin{array}{l}-0.21 \\
(1.02)\end{array}$ & $(-0.62,0.21)$ \\
\hline $\mathrm{HS}$ & 15 & $\begin{array}{c}0.24 \\
(0.96)\end{array}$ & $(-0.25,0.73)$ & $\begin{array}{l}-0.03 \\
(1.16)\end{array}$ & $\begin{array}{r}(-0.54 \\
0.48)\end{array}$ & $\begin{array}{c}0.13 \\
(0.82)\end{array}$ & $(-0.32,0.58)$ & $\begin{array}{c}0.09 \\
(0.93)\end{array}$ & $(-0.43,0.60)$ \\
\hline BA & 19 & $\begin{array}{c}0.32 \\
(0.51)\end{array}$ & $(-0.11,0.76)$ & $\begin{array}{c}0.07 \\
(0.94)\end{array}$ & $\begin{array}{r}(-0.39, \\
0.52)\end{array}$ & $\begin{array}{c}0.22 \\
(0.68)\end{array}$ & $(-0.18,0.62)$ & $\begin{array}{l}-0.13 \\
(1.00)\end{array}$ & $(-0.59,0.32)$ \\
\hline MA & 26 & $\begin{array}{c}0.12 \\
(0.91) \\
\end{array}$ & $(-0.25,0.50)$ & $\begin{array}{c}0.26 \\
(0.80) \\
\end{array}$ & $\begin{array}{r}(-0.13, \\
0.65) \\
\end{array}$ & $\begin{array}{c}0.47 \\
(0.89) \\
\end{array}$ & $(0.13,0.81)$ & $\begin{array}{c}0.23 \\
(1.02) \\
\end{array}$ & $(-0.16,0.62)$ \\
\hline \multicolumn{10}{|c|}{ Labor force status } \\
\hline PLI & 9 & $\begin{array}{l}-0.80 \\
(1.15)\end{array}$ & $\begin{array}{r}(-1.44 \\
-0.16)\end{array}$ & $\begin{array}{l}-0.63 \\
(0.82)\end{array}$ & $\begin{array}{r}(-1.29 \\
0.03)\end{array}$ & $\begin{array}{l}-0.95 \\
(0.96)\end{array}$ & $(-1.58,-0.32)$ & $\begin{array}{l}-0.69 \\
(0.72)\end{array}$ & $(-1.34,-0.03)$ \\
\hline ST & 17 & $\begin{array}{l}-0.28 \\
(0.83)\end{array}$ & $(-0.74,0.19)$ & $\begin{array}{c}0.16 \\
(1.00)\end{array}$ & $\begin{array}{r}(-0.32 \\
0.64)\end{array}$ & $\begin{array}{l}-0.11 \\
(0.89)\end{array}$ & $(-0.57,0.35)$ & $\begin{array}{c}0.20 \\
(0.72)\end{array}$ & $(-0.28,0.68)$ \\
\hline PL & 55 & $\begin{array}{c}0.20 \\
(0.97)\end{array}$ & $(-0.06,0.46)$ & $\begin{array}{c}0.06 \\
(1.02)\end{array}$ & $\begin{array}{r}(-0.21, \\
0.33)\end{array}$ & $\begin{array}{c}0.17 \\
(0.97)\end{array}$ & $(-0.09,0.43)$ & $\begin{array}{c}0.06 \\
(1.09)\end{array}$ & $(-0.21,0.32)$ \\
\hline ws & & 0.205 & & 0.026 & & 0.461 & & 0.017 & \\
\hline \multicolumn{10}{|l|}{ (2) } \\
\hline & & \multicolumn{8}{|c|}{ IA } \\
\hline & & \multicolumn{2}{|c|}{ Learning } & \multicolumn{2}{|c|}{ Physical } & \multicolumn{2}{|c|}{ Creativity } & \multicolumn{2}{|c|}{ Uncertainty } \\
\hline & $N$ & $M(S D)$ & $95 \% \mathrm{CI}$ & $M(S D)$ & $95 \% \mathrm{CI}$ & $M(S D)$ & $95 \% \mathrm{CI}$ & $M(S D)$ & $95 \% \mathrm{CI}$ \\
\hline \multicolumn{10}{|l|}{ Gender } \\
\hline Male & 33 & $\begin{array}{c}0.23 \\
(0.91)\end{array}$ & $(-0.12,0.57)$ & $\begin{array}{c}0.34 \\
(1.01)\end{array}$ & $(0.00,0.67)$ & $\begin{array}{c}0.47 \\
(0.79)\end{array}$ & $(0.15,0.79)$ & $0.23(0.93)$ & $(-0.11,0.58)$ \\
\hline Female & 50 & $\begin{array}{l}-0.15 \\
(1.04)\end{array}$ & $(-0.43,0.13)$ & $\begin{array}{l}-0.22 \\
(0.94) \\
\end{array}$ & $(-0.50,0.05)$ & $\begin{array}{l}-0.31 \\
(1.01)\end{array}$ & $(-0.57,-0.05)$ & $\begin{array}{l}-0.15 \\
(1.02)\end{array}$ & $(-0.43,0.12)$ \\
\hline \multicolumn{10}{|l|}{$\begin{array}{l}\text { Educational } \\
\text { attainment }\end{array}$} \\
\hline LHS & 23 & $\begin{array}{l}-0.42 \\
(1.13)\end{array}$ & $\begin{array}{l}(-0.81 \\
-0.03)\end{array}$ & $\begin{array}{l}-0.34 \\
(0.95)\end{array}$ & $(-0.74,0.05)$ & $\begin{array}{l}-0.42 \\
(1.08)\end{array}$ & $(-0.83,-0.01)$ & $\begin{array}{l}-0.49 \\
(0.92)\end{array}$ & $(-0.88,-0.11)$ \\
\hline HS & 15 & $\begin{array}{l}-0.09 \\
(0.76)\end{array}$ & $(-0.57,0.40)$ & $\begin{array}{c}0.70 \\
(1.05)\end{array}$ & $(0.21,1.19)$ & $\begin{array}{c}0.20 \\
(1.17)\end{array}$ & $(-0.30,0.71)$ & $0.56(1.05)$ & $(0.08,1.04)$ \\
\hline BA & 19 & $\begin{array}{l}-0.15 \\
(1.01)\end{array}$ & $(-0.58,0.28)$ & $\begin{array}{c}0.05 \\
(0.97)\end{array}$ & $(-0.39,0.48)$ & $\begin{array}{c}0.08 \\
(0.74)\end{array}$ & $(-0.37,0.53)$ & $\begin{array}{l}-0.25 \\
(1.02)\end{array}$ & $(-0.67,0.18)$ \\
\hline MA & 26 & $\begin{array}{c}0.54 \\
(0.79)\end{array}$ & $(0.17,0.90)$ & $\begin{array}{l}-0.13 \\
(0.87)\end{array}$ & $(-0.51,0.24)$ & $\begin{array}{c}0.20 \\
(0.93) \\
\end{array}$ & $(-0.18,0.58)$ & $0.29(0.79)$ & $(-0.07,0.66)$ \\
\hline
\end{tabular}


(Table 4 continued)

\begin{tabular}{|c|c|c|c|c|c|c|c|c|c|}
\hline \multicolumn{10}{|c|}{ Labor force status } \\
\hline PLI & 9 & $\begin{array}{l}-0.77 \\
(0.85)\end{array}$ & $(-1.42,-0.12)$ & $\begin{array}{l}-0.54 \\
(0.86)\end{array}$ & $(-1.21,0.13)$ & $\begin{array}{l}-0.93 \\
(1.22)\end{array}$ & $(-1.57,-0.30)$ & $\begin{array}{l}-0.60 \\
(0.82)\end{array}$ & $(-1.24,0.04)$ \\
\hline ST & 17 & $\begin{array}{l}-0.08 \\
(0.86)\end{array}$ & $(-0.55,0.40)$ & $\begin{array}{c}0.13 \\
(0.99)\end{array}$ & $(-0.36,0.61)$ & $\begin{array}{l}-0.14 \\
(0.94)\end{array}$ & $(-0.60,0.32)$ & $\begin{array}{l}-0.45 \\
(0.64)\end{array}$ & $(-0.92,0.01)$ \\
\hline PL & 55 & $\begin{array}{c}0.14 \\
(1.03)\end{array}$ & $(-0.12,0.40)$ & $\begin{array}{c}0.06 \\
(1.03)\end{array}$ & $(-0.21,0.33)$ & $\begin{array}{c}0.18 \\
(0.92)\end{array}$ & $(-0.07,0.44)$ & $\begin{array}{c}0.23 \\
(1.06)\end{array}$ & $(-0.03,0.48)$ \\
\hline$w s$ & & 0.239 & & -0.245 & & 0.422 & & 0.171 & \\
\hline \multicolumn{10}{|l|}{ (3) } \\
\hline & & & \multicolumn{7}{|c|}{ PNS } \\
\hline & & & \multicolumn{4}{|c|}{ PNS_D } & \multicolumn{3}{|c|}{ PNS_R } \\
\hline & $N$ & & $M(S D)$ & & $95 \% \mathrm{CI}$ & & $M(S D)$ & & $95 \% \mathrm{CI}$ \\
\hline \multicolumn{10}{|l|}{ Gender } \\
\hline Male & 33 & & $-0.20(1.08)$ & & $(-0.54,0.15)$ & & $-0.14(0.90)$ & & $(-0.48,0.21)$ \\
\hline Female & 50 & & $0.13(0.93)$ & & $(-0.15,0.41)$ & & $0.09(1.06)$ & & $(-0.19,0.37)$ \\
\hline \multicolumn{10}{|c|}{$\begin{array}{l}\text { Educational } \\
\text { attainment }\end{array}$} \\
\hline LHS & 23 & & $0.59(1.05)$ & & $(0.21,0.98)$ & & $0.46(1.08)$ & & $(0.07,0.86)$ \\
\hline HS & 15 & & $0.08(0.60)$ & & $(-0.39,0.56)$ & & $0.21(0.72)$ & & $(-0.28,0.70)$ \\
\hline BA & 19 & & $-0.19(0.98)$ & & $(-0.62,0.23)$ & & $-0.16(0.78)$ & & $(-0.59,0.27)$ \\
\hline MA & 26 & & $-0.43(0.93)$ & & $(-0.80,-0.07)$ & & $-0.41(1.05)$ & & $(-0.79,-0.04)$ \\
\hline \multicolumn{10}{|c|}{ Labor force status } \\
\hline PLI & 9 & & $0.53(0.98)$ & & $(-0.12,1.19)$ & & $0.71(1.14)$ & & $(0.05,1.37)$ \\
\hline ST & 17 & & $-0.24(1.15)$ & & $(-0.71,0.24)$ & & $-0.15(0.96)$ & & $(-0.63,0.33)$ \\
\hline PL & 55 & & $-0.04(0.93)$ & & $(-0.30,0.23)$ & & $-0.07(0.98)$ & & $(-0.34,0.19)$ \\
\hline
\end{tabular}

Notes. PNS_D: desire for structure; PNS_R: response to lack of structure; ws: coefficients from first standardized discriminate function; CI: confidence interval; LHS: lower than high school degree; HS: high school degree; BA: bachelor; MA: master degree or more; PLI: retired, unemployed, and homemaker; ST: student; PL: professional active.

Significant differences for PNS as a composite score were found for educational attainment, $F_{(3,79)}=0.573$, $p<0.01, \eta^{2}=0.179$, power $=0.939$. Pairwise comparison of the groups revealed that only the LHS group differed significantly from the MA group $\left(z_{\mathrm{LHS}}=0.58 v s . z_{\mathrm{MA}}=-0.47\right)$. Again, it appeared that people with a lower level of education saw themselves as having a stronger need for structure than the group displaying the highest educational attainment. Furthermore, there was no significant difference between the four educational attainment groups for COFL, $F_{(3,79)}=2.45$, $n s$.

Finally, educational groups differed significantly regarding rigidity of attitudes, $F_{(3,79)}=6.68, p<0.01, \eta^{2}$ $=0.202$, and power $=0.968$. Pairwise comparison showed that significant differences appear between the LHS and the BA group $\left(z_{\mathrm{LHS}}=0.58 v s . z_{\mathrm{BA}}=-0.35\right)$, and MA group $\left(z_{\mathrm{LHS}}=0.58 v s . z_{\mathrm{MA}}=-0.44\right)$, but not with the HS group. All univariate analyses pointed in the same direction; that is to say, the higher the educational level of the person and the higher the self-evaluated capacity to adapt, the lower the need for a structured environment, and the less rigid the person evaluated him/herself (see Table 3).

Because results showed that the four educational attainment groups differed significantly by IA, the authors proceeded to compute an MANOVA to analyze the different dimensions of IA (see Table 4). The one-way MANOVA results revealed a significant multivariate main effect for educational attainment, Wilk's $\Lambda$ $=0.44, F_{(24,209.42)}=2.89, p<0.01$, and partial $\eta^{2}=0.242$. Power to detect the effect was 1 . Given the significance of MANOVA, the authors examined the univariate main effects of education and found significant 
differences for crisis, $F_{(3,79)}=3.89, p<0.05$, partial $\eta^{2}=0.129$, power $=0.807:\left(z_{\mathrm{LHS}}=-0.56 v s . z_{\mathrm{BA}}=0.32\right)$; work stress, $F_{(3,79)}=9.49, p<0.05$, partial $\eta^{2}=0.265$, power $=0.996$ : the LHS group was significantly different from all groups ( $z_{\mathrm{LHS}}=-0.80 v$ s. $\left.z_{\mathrm{HS}}=0.13, z_{\mathrm{BA}}=0.22, z_{\mathrm{MA}}=0.47\right)$; learning, $F_{(3,79)}=4.55, p<0.01$, partial $\eta^{2}=0.147$, power $=0.871:\left(z_{\mathrm{LHS}}=-0.42 v s . z_{\mathrm{MA}}=0.54\right)$; physical, $F_{(3,79)}=3.85, p<0.05$, partial $\eta^{2}=$ 0.128 , power $=0.804:\left(z_{\mathrm{LHS}}=-0.34 v s . z_{\mathrm{HS}}=0.70\right)$; and uncertainty, $F_{(3,79)}=5.25, p<0.01$, partial $\eta^{2}=0.166$, power $=0.917$ : The LHS group was different from the HS and the MA but not from the BA group $\left(z_{\mathrm{LHS}}=-0.49\right.$ vs. $z_{\mathrm{HS}}=0.56, z_{\mathrm{BA}}=0.29$ ).

In regard to the PNS scale, the two components were included in an MANOVA. The estimated value for Wilk's $\Lambda$ was $0.806, F_{(6,156)}=2.97, p<0.01$, partial $\eta^{2}=0.102$, and power to detect the effect was .894 , which revealed a significant effect of education on PNS. Significant univariate main effects for this scale were obtained for both components, desire for structure, $F_{(3,79)}=5.29, p<0.01$, partial $\eta^{2}=0.167$, power $=0.919$ : LHS differed significantly from the BA and the MA group $\left(z_{\mathrm{LHS}}=0.59 v s . z_{\mathrm{BA}}=-0.19, z_{\mathrm{MA}}=-0.43\right)$; and response to lack of structure, $F_{(3,79)}=3.88, p<0.05$, partial $\eta^{2}=0.128$, power $=0.806$ : LHS group was significantly different from the MA group $\left(z_{\mathrm{LHS}}=0.46 v s . z_{\mathrm{MA}}=-0.41\right)$.

Educational attainment accounted for about $20 \%$ of the variance surrounding the overall IA composite score, showing that lower educated subjects tended to attribute themselves lower scores on their overall capacity to adapt than higher educated subjects. Even though this difference was not found for COFL, similar results were obtained for PNS (17.9\% of explained variance) and rigidity of attitudes $(20.2 \%$ of explained variance) where lower educated subjects saw themselves as more rigid and as having a stronger need for structured environments than higher educated subjects.

Educational attainment explained $24.2 \%$ of the variance in the various dimensions of IA. Education is importantly associated with the work stress dimension ( $26.5 \%$ explained variance) followed by the uncertainty $(16.6 \%)$ and the learning dimensions $(14.7 \%)$, and finally the crisis and the physical dimensions $(12.9 \%$ and $12.8 \%$, respectively). Accordingly, persons that had lower education evaluated themselves as having lower competences in dealing with work stress situations (i.e., high workload, overtime) and having more difficulties when confronted with unstable and changing situations than higher educated persons. Significant differences for rigidity and both components of PNS were also found (16.7\% and 12.8\% of explained variances for the desire and the response component, respectively), which indicated that lower educated subjects reported having a higher desire for structure and tended to respond to lack of structure more strongly than subjects that had a higher educational degree.

From the current results, it can only be stated that educational attainment was associated with differences in self-perceived level in the capacity to adapt to changing situations. That is to say, from these results, it cannot be known that if persons can increase or have increased their adaptability by studying, or whether the more adaptable they are, the higher levels of education they attain.

\section{Labor Force Status}

The authors also analyzed differences between groups according to their current situation. The authors compared three groups: ST (students) $(N=17)$, PL (professionals active) $(N=55)$, and PLI (professionally inactive) people like homemakers, retired persons, or unemployed $(N=9)$; it did not have indication about the current situation for two persons. Differences in IA, PNS, COFL, and rigidity of attitudes were all explored with ANOVAs. 
The authors found significant differences for IA, $F_{(2,78)}=7.45, p<0.01, \eta^{2}=0.160$, power $=0.934$. Pairwise comparison (see Table 3) showed the differences were due to a significant disparity between PL and PLI persons $\left(z_{\mathrm{PL}}=0.21 v s . z_{\mathrm{PLI}}=-1.08\right)$. The authors found no significant effect of the labor force status for PNS, $F_{(2,78)}=2.91, n s$. A significant difference for the labor force status variable appeared for COFL, $F_{(2,78)}=$ $4.20, p<0.05, \eta^{2}=0.097$, power $=0.722$ : significant differences exist only between the PL and PLI groups $\left(z_{\mathrm{PL}}\right.$ $\left.=0.18 v s . z_{\mathrm{PLI}}=-0.81\right)$. Finally, the labor force status was also related to rigidity of attitudes, $F_{(2,78)}=3.15, p<$ $0.05, \eta^{2}=0.075$, power $=0.588$. The difference found here was between the ST and the homemakers $\left(z_{\mathrm{ST}}=\right.$ -0.24 vs. $z_{\mathrm{PLI}}=0.75$ ).

Because the ANOVA analyses showed that the labor force status appeared to have an effect on IA, the authors continued the investigation by computing an MANOVA for the different related dimensions of the IA scale. The MANOVA revealed a significant multivariate main effect for the labor force status variable, Wilk's $\Lambda=0.685, F_{(16,142)}=1.84, p<0.05$, partial $\eta^{2}=0.172$, power $=0.936$. The univariate analyses showed that the significant effects for labor force status was only for crisis, $F_{(2,78)}=4.96, p<0.01$, partial $\eta^{2}=0.113$, power to detect $=0.796$ : significant difference was found for the PL and PLI group $\left(z_{\mathrm{PL}}=0.20 v\right.$ s. $\left.z_{\mathrm{PLI}}=-0.80\right)$; work stress, $F_{(2,78)}=5.42, p<0.01$, partial $\eta^{2}=0.122$, power $=0.832$ : differences were found for the PL and PLI group $\left(z_{\mathrm{PL}}=0.17\right.$ vs. $\left.z_{\mathrm{PLI}}=-0.95\right)$; learning, $F_{(2,78)}=3.40, p<0.05$, partial $\eta^{2}=0.080$, power $=0.623$ : the PL and PLI group appeared to be significantly different $\left(z_{\mathrm{PL}}=0.14 v\right.$ s. $\left.z_{\mathrm{PLI}}=-0.77\right)$; creativity, $F_{(2,78)}=5.47, p<$ 0.01 , partial $\eta^{2}=0.123$, power $=0.836$ : significant differences were found for the PL and PLI group $\left(z_{\mathrm{PL}}=0.18\right.$ vs. $\left.z_{\mathrm{PLI}}=-0.93\right)$; uncertainty, $F_{(2,78)}=5.12, p<0.01$, partial $\eta^{2}=0.116$, power $=0.809$ : the ST group was significantly different from the PL group $\left(z_{\mathrm{ST}}=-0.45 v s . z_{\mathrm{PL}}=0.23\right)$, but the differences between the PL group and the PLI group were similar $\left(z_{\mathrm{PL}}=0.23 v s . z_{\mathrm{PLI}}=-0.60, p=0.058\right)$. Moreover, no significant differences were found between the groups for both components of PNS.

The current labor force status of being a student, having a job, or staying at home seemed to be related to overall IA ( $16 \%$ of explained variance), COFL ( $9.7 \%$ of explained variance), and rigidity of attitudes ( $7.5 \%$ of explained variance). In regard to IA, COFL, and rigidity, all the differences that were described, persons that were not PL (homemaker, retired, and unemployed) attributed themselves lower scores for IA and COFL and higher scores for rigidity of attitudes. At a dimensional level, $12.3 \%$ of the variance was explained by the labor force status in creativity and $12.2 \%$ in work stress, followed by uncertainty and the crisis dimension (11.6\% and $11.3 \%$, respectively), and finally, the learning dimension (8\%). The professionally inactive group was found to have lower scores than the PL and ST groups; differences varied by dimensions (see Table 4).

To gain better insight on how these groups may be differentiated, the authors adopted a multivariate combination approach for the different dimensions of IA. Consequently, the authors followed Grice and Iwasaki (2007) and conducted a one-factor and between-subjects multivariate analysis of variance. This approach is interesting, because it allows us to understand how people differentiate at once on all eight dimensions combined instead of considering the dimensions one by one, or two at a time. As all adaptability dimensions are related, it seems reasonable to attempt to distinguish persons on this combination of dimensions. The authors choose to use this method to compare means in regard to the labor force status as it might be useful to have this complementary information when evaluating persons for career development. As all IA dimensions are related (see Table 5), performing a multivariate combination of IA sub-scales made sense. 
Table 5

Correlations Between IA Dimensions

\begin{tabular}{|c|c|c|c|c|c|c|c|c|}
\hline & \multicolumn{8}{|c|}{ IA } \\
\hline & Crisis & Culture & $\begin{array}{l}\text { Work } \\
\text { stress }\end{array}$ & Inter-personal & Learning & Physical & Creativity & Uncertainty \\
\hline Crisis (1) & - & & & & & & & \\
\hline Culture (2) & $0.46^{* *}$ & - & & & & & & \\
\hline Work stress (3) & $0.59^{* *}$ & $0.23^{*}$ & - & & & & & \\
\hline Inter-personal (4) & $0.30^{*}$ & $0.51^{* *}$ & 0.09 & - & & & & \\
\hline Learning (5) & $0.34^{* *}$ & $0.32^{* *}$ & $0.24^{*}$ & $0.50^{* *}$ & - & & & \\
\hline Physical (6) & $0.48^{* *}$ & $0.45^{* *}$ & $0.43^{* *}$ & $0.22^{*}$ & 0.11 & - & & \\
\hline Creativity (7) & $0.55^{* *}$ & $0.35^{* *}$ & $0.41^{* *}$ & $0.47^{* *}$ & $0.48^{* *}$ & $0.44^{* *}$ & - & \\
\hline Uncertainty (8) & $0.54^{* *}$ & $0.39^{* *}$ & $0.59^{* *}$ & $0.30^{*}$ & $0.34^{* *}$ & $0.44^{* *}$ & $0.45^{* *}$ & - \\
\hline
\end{tabular}

Notes. ${ }^{* *} p<0.01 ;{ }^{*} p<0.05$.

Results from MANOVA were statistically significant, Wilk's $\Lambda=0.685, F_{(16,142)}=1.84, p<0.05, \eta^{2}=$ 0.172 , power $=0.936$. Univariate means, standard deviations, and the $S D$ function coefficients (ws) for the first multivariate combination are reported in Table 4. Differences were significant for the overall test $F_{(2,78)}=9.35$, $p<0.01, \eta^{2}=0.193$. Follow-up contrast showed that all three groups differed significantly on the multivariate combination $\left(z_{\mathrm{PLI}}=-1.18 v\right.$ s. $z_{\mathrm{ST}}=-0.29$ vs. $\left.z_{\mathrm{PL}}=0.26, p<0.05\right)$. PLI scored even lower on average than ST who scored lower than PL (see Table 6 and Figure 1).

Table 6

Standardized Means (Z-scores) and SD for Multivariate Combination of IA Dimensions

\begin{tabular}{llc}
\hline Labor force status & \multicolumn{2}{c}{ Multivariate combination } \\
\cline { 2 - 3 } & $M(S D)$ & $95 \% \mathrm{CI}$ \\
\hline PLI & $-1.18(0.99)$ & $(-1.94,-0.42)$ \\
ST & $-0.29(0.70)$ & $(-0.65,0.07)$ \\
PL & $0.26(1.02)$ & $(-0.02,0.53)$ \\
\hline
\end{tabular}

Notes. CI: confidence interval; PLI: retired, unemployed, and homemaker; ST: student; PL: professional active.

The multivariate analyses were more informative as the authors were able to distinguish between the three groups, which was not a possibility via the univariate pairwise comparison following the MANOVA. As the different dimensions were related, it made sense to analyze differences between groups based on their combination and not on individual dimensions. Interestingly, the three groups were found to be different only in regard to the strength of the scores for the various dimensions and not in their direction. Note that similar to educational attainment, it cannot be said if persons that perceive themselves as more adaptable are more likely to study, or in this case, are more likely to be employed; or if being part of the professional world has a positive influence on how adaptable people perceive themselves. That is to say, it is not known if the context (PLI, ST, and PL) leads to higher self-perceived adaptability or if adaptability leads to a given context of labor force status.

Returning to the analysis, it should be noted that no significant MANOVA interaction effects are found for the three independent variables (gender, educational attainment, and labor force status). 


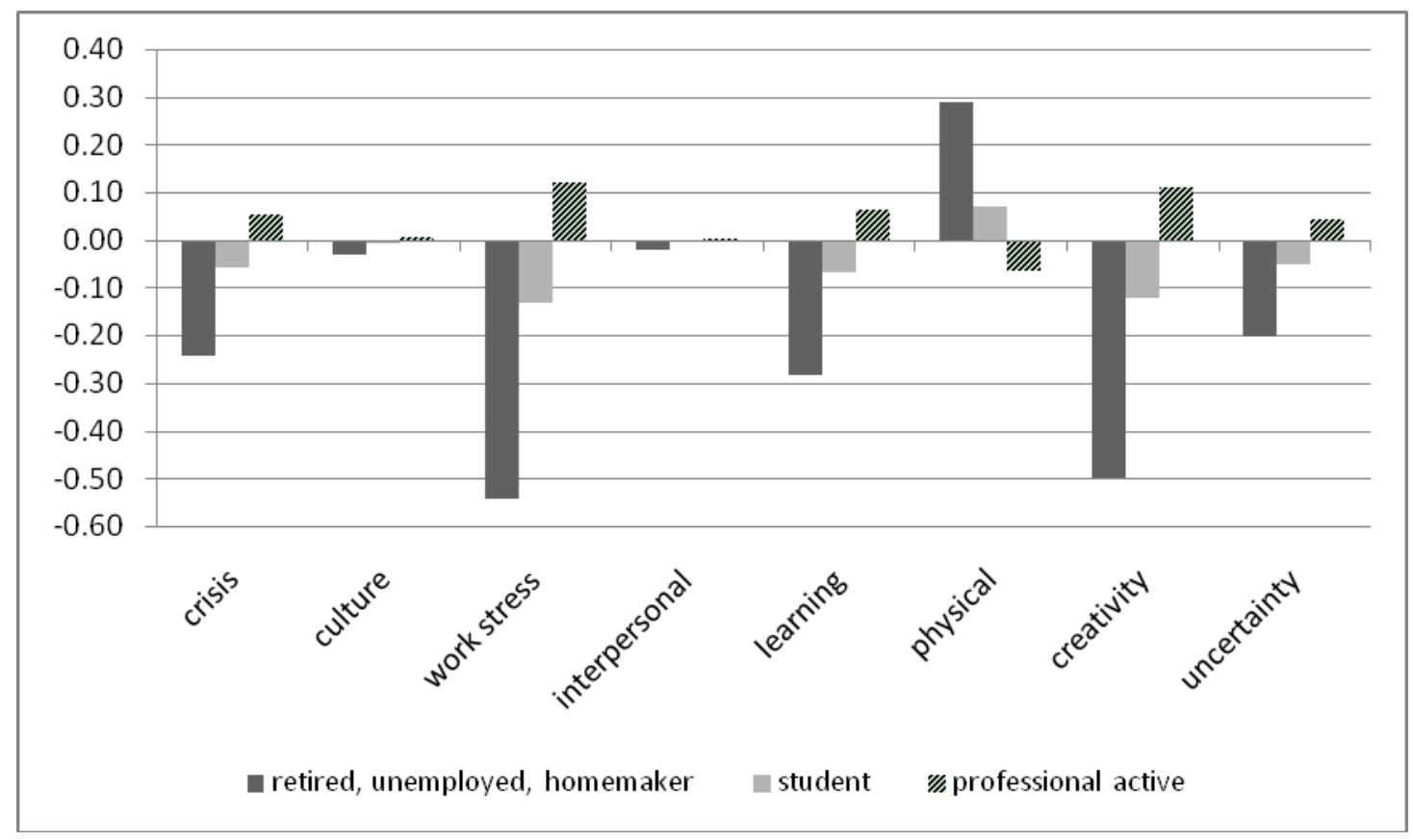

Figure 1. Each group on the multivariate combination of IA dimensions.

\section{Discussion and Conclusions}

The goal of this paper was to investigate IA together with potentially related constructs. Therefore, the authors analyzed convergent and divergent validity by examining adaptability relative to rigidity of attitudes, PNS, and COFL. The authors explored the relations between the concepts using correlational analyses (correlations and regressions). The person's willingness and the belief in his/her self-efficacy for being flexible appear strongly related to overall IA as well as to its dimensions. Even though the correlation between COFL and the creativity dimension appeared to be higher than the other correlations, they were not significantly different. Nevertheless, the large proportion of variance accounted for IA by COFL is an argument in favor of convergent validity.

The regression analysis also reveals that $47 \%$ of the overall IA can be accounted for by COFL and the "desire for structure" component of the PNS scale. It seems that people who evaluate themselves as being adaptable are also persons that see themselves as being able and willing to be flexible and whose desire for structure is low. COFL as defined by Martin and Rubin (1995) appeared to play an important role for all dimensions of adaptability. As such, this concept appears to be importantly related to adaptability. An awareness for different situations and willingness to react in a flexible way combined with a belief in one's own self-efficacy essentially appears to reflect the persons' capacity to adapt. Interestingly, COFL was retained as a predictor for all dimensions of IA while rigidity of attitudes and PNS had lower predictive power, and only for some of the dimensions. Thus, it appears that being cognitively flexible is importantly associated with adaptability, but not sufficient to explain the entire concept.

The cultural dimension involved COFL and rigidity of attitudes while the work stress, physical, and uncertainty dimensions relied on COFL and the desire for structure component of the PNS scale. The cultural dimension of IA asks candidates about their capacity to cope when confronted with persons having different cultural backgrounds and having values and sets of beliefs that differ from their own. Personal habits, beliefs, 
and values may be the grounds that relate this dimension to rigidity of attitudes. It is reasonable that a person who is less open to new experiences needs familiar and systematic behavioral patterns, and thus, has more difficulties accepting people who do not fit into his/her structured habitual environment. As such, unknown values and habits appear to be destabilizing and are not desirable. Furthermore, uncertain situations, unstable and stressful work situations, as well as difficult environmental conditions may be hard to manage for people that have a high desire for structure. Therefore, a combination of being able to deal with complex problems and unstructured environments is associated with the uncertainty, work stress, and physical dimensions of IA.

Moreover, correlations were not significantly different for both components of PNS with adaptability and were similar for both components with rigidity $(r=0.60, p<0.01$ and $r=0.58, p<0.01)$. Thus, it seems that PNS has more in common with rigidity of attitudes and personal habits (Steinmetz et al., 2011) than with IA. This finding is in support of divergent validity for the IA scale. As such, even though the PNS, calling for routinized and well-organized environments, is moderately related to the person's capacity to adapt, the authors believe that IA allows for a more nuanced evaluation of the person's capacity to deal with changing situations in regard to various contexts (through the different dimensions) than the PNS scale.

Finally, the rigidity concept appears to be mostly related to the work stress and the uncertainty dimensions of IA. This makes good sense as the rigidity scale evaluates how rigid or flexible people judge themselves in different situations regarding their personal habits. As rigid people tend to prefer systematic and familiar environments, it is not surprising that unmanageable workload, breaking down systematic working behavior, or unpredictable and uncertain circumstances are perceived as uncomfortable and unpleasant. In the stepwise linear regression model, rigidity remained a predictor only for the cultural dimension of adaptability, which can be interpreted as resulting from the rigid person's inflexible attitude in regards to his/her own differing values. This result corroborates Steinmetz et al.'s result (2011), where the personality trait "openness" from the NEO (neuroticism-extroversion-openness) Five Factor Inventory (McCrae \& Costa, 1987) was correlated at $r=-0.44$, $p<0.05$ with rigidity of attitudes regarding personal habits. Suggesting that rigid people are less open to new experiences, and they are also less open to people having different cultural origins and values.

Given that the crisis, interpersonal learning, and creativity dimensions may be associated with COFL, but the physical, work stress, uncertainty, and culture dimensions are also associated with PNS and rigidity, the authors believe that this provides support for the multi-dimensionality of IA. That is to say, the differential relationships suggest that "COFL" is important for every IA dimension; however, the different dimensions also contain separate specific information that correlates with rigidity of attitudes or PNS.

To sum up the first part of our study, the authors contend that IA involves COFL as evaluated by Martin and Rubin's (1995) scale, but it is also related to PNS and to some extent to rigidity. Nevertheless, the authors remind the reader that they rely exclusively on self-assessment instruments and therefore depend greatly on the subject's ability to accurately self-assess.

In the second part of this paper, the authors presented individual differences based on socio-demographic variables. The authors find gender difference in how adaptable persons perceived themselves to be. Men evaluate themselves as more adaptable than women do, particularly in regard to work stress and in finding creative solutions for complex problems. Because the authors use self-evaluations, it is impossible to say if men and women are really different in regards to some of these dimensions of adaptability, or if they simply perceive themselves to be different. As noted by Beyer (1990), expectations are involved in accuracy of self-evaluations for men and women. Accordingly, women tend to be more modest than men when self-evaluating 
their performance, especially when they have to evaluate their performance on what is thought to be masculine tasks; whereas they appear to report less modestly regarding their performance on "feminine" tasks.

Since men and women differ most importantly on the work stress dimension and problem-solving, which arguably have masculine connotations, the differences may be due to bias in self-evaluations. A meta-analytic study by Jick and Mitz (1985) provides some support for the more masculine connotation of the work stress dimension; they show that women tend to report stress more often related symptoms at work, such as low subjective well-being than men. However, men often have more stress related health problems, such as coronary heart disease than women. These findings corroborate our results in that men seem to perceive themselves as having higher capacities for dealing with work stress than women. Women may be more susceptible to early stress symptoms than men and thus report lower capacities for dealing with work stress.

Similar to work stress, it appears that men report higher confidence in their problem-solving capacity than women. Here, again the authors suspect a masculine connotation effect on self-perceived problem-solving capacity, as the literature examples show that boys have higher expectations in regard to their reasoning capacities than girls and that parents tend to expect better mathematical performance from boys than from girls (Eccles \& Jacobs, 1986). It is possible that men have a more positive view of their own problem-solving capacities than women, based on their own past experience. Thus, even though differences appear to be significantly different for these dimensions, our findings do not allow us to conclude whether the differences are real objective differences based on accurate judgments or if the evaluations are biased. Regardless of the higher of the scores for men over women, the crucial message is that these scales differentiate between men and women and therefore should not be used to compare adaptability level of men and women in selection procedures. The fact that on average women report lower adaptability competences than men should not be interpreted as women being less adaptable than men, and thus the scale should not be used to make selection decisions between men and women. Interestingly, however, no gender related differences were detected for rigidity and PNS; again confirming that IA as measured by this scale is different from these two constructs.

The authors also find that educational attainment is associated with how people perceive themselves in reference to adaptability. Overall, it appears that participants with higher education report higher levels of IA, lower levels of rigidity, and less PNS. These results, however, should be accepted with some caution. It is of high importance to understand that these results do not infer any causality. Moreover, it should be aware that these self-reports might be biased by the participants' language skills as a consequence of their educational level. From the current results, we can only state that educational attainment is associated with differences in the self-perceived level of adaptability, but the question of whether persons become more adaptable by pursuing higher education or whether the more adaptable people are more likely to pursue higher education is unable to be answered.

This issue gets at the core of adaptability. If adaptability is to be seen as a trait then probably being more adaptable is a useful characteristic for engaging in education. However, if adaptability is a capacity that can be acquired and developed, then education may have an influence on how adaptable a person is or perceives him/herself to be. Ployhart and Bliese (2006) avoided a concrete answer to this question when positioning IA on a distal-proximal continuum on which IA can be seen as more trait-like (Ployhart \& Bliese, 2006, p. 17). Where trait-like is thought to be relatively stable and enduring, not much affected by situations or experience. For example, KSAOs are described to be distal predictors that are fairly stable and trait-like. On the same continuum, IA is neighboring personality, cognitive ability, interest, and other KSAOs, but it is depicted as 
being closer (e.g., compared to personality) to proximal predictors that are more state-like (i.e., performance) and therefore somewhat more malleable. Based on Ployhart and Bliese's (2006) configuration, the findings could be interpreted as saying that adaptable subjects tend to attain higher levels of education. However, in the same breath, I-ADAPT theory claims that IA is determined to some degree by KSAOs like personality, cognitive ability, and interests. Thus, it cannot be seen as absolutely stable.

If adaptability was not to be absolutely stable, this could be important in the frame of a possible intervention for organizations that plan to reorganize. It would mean that persons who are evaluated to be presently less adaptable to change could benefit from "adaptability-training", which could lead to better outcomes when faced with coming change and/or transitions. Even though adaptability should not be considered as isolated measure but rather combined with other measures and interviews, it could be seen as extremely important in the professional context. Therefore, the present study included analyses based on differences between groups that were classified according to their labor force status. PL, ST, and PLI people were compared in regard to IA, COFL, PNS, and RAPH. The groups differed in their capacity to adapt, in their level of COFL and also in their level of rigidity, but not in their PNS. Generally, professionally active persons appeared to see themselves as less adaptable, less cognitively flexible than professionally active persons and more rigid in their attitudes than ST.

Results from the one-factor MANOVA revealed homemakers were always lower than ST and PL; and ST were lower than PL for the combination of IA dimensions. Again, these results have to be interpreted with caution. The authors strongly believe that the measures presented here should be used for profiling in order to promote the personal and professional development of the persons. But, the current results should not lead to the stigmatization based on labor force status groups, rather results should inform how the different groups typically perceive themselves in their everyday context. This is of high importance because results do not allow us to infer a causal relationship. As such, it is unable to tell if the labor force status leads to a given level of adaptability or rigidity; or if being low on adaptability and high on rigidity leads to given life circumstances. This argument is especially important in regard to the homemakers, as results show a relation between this category and lower scores on adaptability and higher scores on rigidity.

Moreover, pointing out the differences between ST and PL should provide valuable information for human resource managers, as they might be confronted to choose between qualified ST and PL. If adaptability was to be developed over time and maybe with experience, professionals would consistently report higher levels of adaptability and lower levels of rigidity compared to ST when considering PL settings. Given that case, IA, COFL, and RAPH should be used only with caution and taking into account group differences.

Accordingly, this research only highlights that these three groups of persons might report different levels of adaptability and that this information ought to be considered. As such, these groups may declare a level of adaptability that has to be understood in light of the day-to-day context they live in.

To conclude, IA is to be considered as a valid construct that is related to, but different from, concepts like rigidity, PNS, and COFL. Because the various IA dimensions are differentially related to COFL, rigidity, and PNS, it makes sense to continue thinking about adaptability as a multi-dimensional construct. Moreover, in reference to COFL, the proportion of additionally explained variance in the different dimensions of IA is quite low, for rigidity and for PNS, thus, the authors suggest these concepts are fairly different from IA and provide further information about the person. Finally, the results show that important differences in adaptability scores for several socio-demographic variables and thus the authors reiterate that it is important to be mindful when 
using these scales, not only in regards to the specific groups the authors investigated, but also when using in a practical context like counseling.

\section{Limitations}

The sample size was fairly small and therefore results have to be interpreted with caution. However, as this is the first attempt to combine adaptability with conceptually related constructs, the authors believe that results are indicative and worth being presented. Moreover, power analysis showed that the power to detect effects was strong enough to trust our findings.

The small sample size also affected the different proportions of groups that were used to compare means. Consequently, in regard to gender, educational attainment, and labor force status, the sample was not balanced. But as stated earlier, the authors contend that our results are of interest and should be considered. Future replications of the study should provide even more clarity on the topic and contribute to generalizability.

Another shortcoming of this research was the poor reliabilities for the interpersonal and the physical dimensions of IA. Weak reliabilities could lead to erroneous conclusions about these dimensions and therefore results concerned with these sub-scales need to be considered with caution. However, previous research showed acceptable reliability for interpersonal dimension and gave reasonable explanation for the low reliability of the physical IA scale.

Furthermore, it needs to be said that the one-factor, between-subjects multivariate analysis of variance that was used in regard to labor force status, also has to be interpreted with caution. According to Stevens (2002), results for discriminate functions are not stable for small samples.

\section{Outlook}

Future research should replicate this study using a larger sample, enabling the researchers to use structural equation modeling to more accurately explore the relations between the different scales. Using a multi-trait or multi-method approach would also provide information about how the concepts are related controlling for the method. Relatedly, it might be of interest not relying exclusively on self-reported measures for the different concepts but on objective and/or behavioral evaluations as suggested by authors like Cañas et al. (2003), Lang and Bliese (2009), or LePine, Colquitt, and Erez (2000) for COFL and adaptability.

Moreover, future research could include a traditional exploratory principal component analysis on an item level to help clarify the relations between the scales and shed light on possible underlying factors in regard to the different constructs (IA, RAPH, PNS, and COFL).

Furthermore, it would be worth analyzing differences between populations. As in the current research, IA appeared to differentiate between groups (gender, educational attainment, and labor force status), it would be important to verify if these are stable differences over time and groups, or if these results were specific to our sample. This would be important especially in regard to gender, but also in regard to education and labor force status. For gender, it would mean that this scale is not be used to compare men and women, but for education and labor force, it would mean that future investigations should attempt to clarify if adaptability is part of the individual (trait) or if adaptability is acquired with education and professional experience. Longitudinal studies would constitute a good opportunity to respond to this question.

Moreover, in regard to experience, it would be interesting to study adaptability, COFL, PNS, and rigidity in relation to age to explore how these concepts develop and evolve over the life course. 


\section{References}

Beyer, S. (1990). Gender differences in the accuracy of self-evaluations of performance. Journal of Personality and Social Psychology, 59, 960-970. doi: 10.1037/0022-3514.59.5.960

Cañas, J., Quesada, J., Antolí, A., \& Fajardo, I. (2003). Cognitive flexibility and adaptability to environmental changes in dynamic complex problem-solving tasks. Ergonomics, 46, 482-501. doi: 10.1080/0014013031000061640

Chen, P. Y., \& Popovich, P. M. (2002). Correlation: Parametric and nonparametric measures. Thousand Oaks, C. A.: Sage.

Cohen, J. (1992). A power primer. Psychological Bulletin, 112, 155-159. doi: 10.1037/0033-2909.112.1.155

Eccles, J. S., \& Jacobs, J. E. (1986). Social forces shape math attitudes and performance. Signs: Journal of Women in Culture and Society, 11, 367-380. doi: 10.1086/494229

Grice, J. W., \& Iwasaki, M. (2007). A truly multivariate approach to MANOVA. Applied Multivariate Research, 12, $199-226$.

Jick, T. D., \& Mitz, L. F. (1985). Sex differences in work stress. The Academy of Management Review, 10, 408-420. doi: $10.2307 / 258124$

Kobasa, S. C. (1979). Stressful life events, personality, and health: An inquiry into hardiness. Journal of Personality and Social Psychology, 37, 1-11. doi: 10.1037//0022-3514.37.1.1

Lang, J. W., \& Bliese, P. (2009). General mental ability and two types of adaptation to unforeseen change: Applying discontinuous growth models to the task-change paradigm. Journal of Applied Psychology, 94, 411-428. doi: 10.1037/a0013803

LePine, J. A., Colquitt, J. A., \& Erez, A. (2000). Adaptability to changing task contexts: Effects of general cognitive ability, conscientiousness, and openness to experience. Personnel Psychology, 53, 563-593. doi: 10.1111/j.1744-6570.2000.tb00214.x

Martin, M. M., \& Rubin, R. B. (1995). A new measure of cognitive flexibility. Psychological Reports, 76, 623-626. doi: 10.2466/pr0.1995.76.2.623

McCrae, R. R., \& Costa, P. T. (1987). Validation of the five-factor model of personality across instruments and observers. Journal of Personality and Social Psychology, 52, 81-90. doi: 10.1037/0022-3514.52.1.81

Meresko, R., Rubin, M., Shontz, F. C., \& Morrow, W. R. (1954). Rigidity of attitudes regarding personal habits and its ideological correlates. Journal of Abnormal and Social Psychology, 49, 89-93. doi: 10.1037/h0056505

Morrison, R. F., \& Hall, D. T. (2002). Career adaptability. In D. T. Hall (Ed.), Careers in and out of organizations (pp. 205-234). Thousand Oaks, C. A.: Sage.

Neuberg, S. L., \& Newsom, J. T. (1993). Personal need for structure: Individual differences in the desire for simple structure. Journal of Personality and Social Psychology, 65, 113-131. doi: 10.1037/0022-3514.65.1.113

O'Connell, D. J., McNeely, E., \& Hall, D. T. (2008). Unpacking personal adaptability at work. Journal of Leadership \& Organizational Studies, 14, 248-259. doi: 10.1177/107191907311005

Ployhart, R. E., \& Bliese, P. D. (2006). Individual adaptability (I-Adapt) theory: Conceptualizing the antecedents, consequences, and measurement of individual differences in adaptability. In S. C. Burke, L. G. Pierce, \& E. Salas (Eds.), Understanding adaptability: A prerequisite for effective performance within complex environments (Vol. 6: Advances in human performance and cognitive engineering research, pp. 3-39). Bingley, U. K.: Emerald.

Pulakos, E. D., Arad, S., Donovan, M. A., \& Plamondon, K. (2000). Adaptability in the workplace: Development of a taxonomy of adaptive performance. Journal of Applied Psychology, 85, 612-624. doi: 10.1037/0021-9010.85.4.612

Pulakos, E. D., Dorsey, D. W., \& White, S. S. (2006). Adaptability in the workplace: Selecting an adaptive workforce. In S. C. Burke, L. G. Pierce, \& E. Salas (Eds.), Understanding adaptability: A prerequisite for effective performance within complex environments (Vol. 6: Advances in human performance and cognitive engineering research, pp. 41-71). Bingley, U. K.: Emerald.

Pulakos, E. D., Schmitt, N., Dorsey, D. W., Arad, S., Borman, W. C., \& Hedge, J. W. (2002). Predicting adaptive performance: Further tests of a model of adaptability. Human Performance, 15, 299-323. doi: 10.1207/S15327043HUP1504_01

Savickas, M. L. (1997). Career adaptability: An integrative construct for life-span, life-space theory. The Career Development Quarterly, 45, 247-259.

Savickas, M. L., Nota, L., Rossier, J., Dauwalder, J. P., Duarte, M. E., Guichard, J., .. Van Vianen, A. (2009). Life designing: A paradigm for career construction in the 21 st century. Journal of Vocational Behavior, 75, 239-250. doi: 10.1016/j.jvb.2009.04.004 
Schultz, P. W., \& Searleman, A. (2002). Rigidity of thought and behavior: 100 years of research. Genetic, Social \& General Psychology Monographs, 128, 165-207.

Steinmetz, J. P., Loarer, E., \& Houssemand, C. (2011). Rigidity of attitudes and behaviors: A study on the validity of the concept. Individual Differences Research, 9, 84-106.

Stevens, J. (2002). Applied multivariate statistics for the social sciences. Mahwah, New Jersey: Lawrence Erlbaum.

Trites, D. (1960). Adaptability measures as predictors of performance ratings. Journal of Applied Psychology, 44, 349-353. doi: $10.1037 / \mathrm{h} 0042148$

Trites, D., Kubala, A., \& Cobb, B. (1959). Development and validation of adaptability criteria. Journal of Applied Psychology, 43, 25-30. doi: 10.1037/h0039879

Tucker, J. S., Pleban, R. J., \& Gunther, K. M. (2010). The mediating effects of adaptive skill on values-performance relationships. Human Performance, 23, 81-99. doi: 10.1080/08959280903400275

Wessel, J., Ryan, A., \& Oswald, F. (2008). The relationship between objective and perceived fit with academic major, adaptability, and major-related outcomes. Journal of Vocational Behavior, 72, 363-376. doi: 10.1016/j.jvb.2007.11.003

\section{Appendix}

Description of the Eight Individual Adaptability Dimensions (Ployhart \& Bliese, 2006; Pulakos et al., 2000)

\begin{tabular}{|l|l|}
\hline Dimension & Description \\
\hline Crisis & Concerned with emergency situations, how people deal with emergency situations \\
\hline Creativity & Concerned with being able to deal with and solve complex problems \\
\hline Cultural & Concerned with accepting and dealing with people having different cultural backgrounds \\
\hline Interpersonal & Concerned with the flexibility and understanding in the interaction with other persons \\
\hline Learning & $\begin{array}{l}\text { Concerned with being interested and willing to learn new skills and competences to be on top of the } \\
\text { job requirements }\end{array}$ \\
\hline Physical & $\begin{array}{l}\text { Concerned with the physical condition (strength and state) of the person but also with the } \\
\text { environmental condition }\end{array}$ \\
\hline Work stress & $\begin{array}{l}\text { Concerned with the amount of work to do, to handle difficult work situations in relation to large } \\
\text { work demand }\end{array}$ \\
\hline Uncertainty & $\begin{array}{l}\text { Concerned with being able to deal with uncertain, changing situations, deal with unpredictable } \\
\text { situations/conditions }\end{array}$ \\
\hline
\end{tabular}

